INPLASY

PROTOCOL

To cite: Zhang et al.

Effectiveness of nonpharmacological interventions for treating post-stroke depression: study protocol of an umbrella review of

systematic reviews and metaanalyses. Inplasy protocol 2021100083. doi:

10.37766/inplasy2021.10.0083

Received: 22 October 2021

Published: 22 October 2021

Corresponding author:

Yuejuan Zhang

1171825297@qq.com

Author Affiliation:

Hunan University of Chinese Medicine.

Support: Hunan Provincial Project.

Review Stage at time of this submission: Preliminary searches.

Conflicts of interest: None declared.

\section{Effectiveness of non-pharmacological interventions for treating post-stroke depression: study protocol of an umbrella review of systematic reviews and meta-analyses}

\author{
Zhang, C1; He, L2; Li, Z3; Qiu, H4; Wang, X5; Zhang, Y6.
}

Review question / Objective: Non-pharmacological therapies have been used for the treatment of post-stroke depression (PSD), and there have been many systematic reviews and meta-analyses evaluating the effectiveness of these nonpharmacological treatments. They help to improve symptoms of PSD and reduce morbidity and mortality in patients with PSD, however, the credibility of the evidence has not been assessed. The purpose of this study was to summarize and evaluate the current evidence for non-pharmacological treatment of PSD and to determine treatment based on reliable evidence.

Condition being studied: Post-stroke depression (PSD) is a common complication of stroke, occurs in nearly a third of stroke patients by a study with a five-year follow-up study. In addition, depression often occurs in the first year after stroke, and patients with prior depression or severe stroke are at higher risk for depression. PSD not only increases the suffering of patients, but also imposes a heavy financial burden on families and society.

INPLASY registration number: This protocol was registered with the International Platform of Registered Systematic Review and Meta-Analysis Protocols (INPLASY) on 22 October 2021 and was last updated on 22 October 2021 (registration number INPLASY2021100083).

\section{INTRODUCTION}

Review question / Objective: Nonpharmacological therapies have been used for the treatment of post-stroke depression (PSD), and there have been many systematic reviews and meta-analyses evaluating the effectiveness of these non- pharmacological treatments. They help to improve symptoms of PSD and reduce morbidity and mortality in patients with PSD, however, the credibility of the evidence has not been assessed. The purpose of this study was to summarize and evaluate the current evidence for nonpharmacological treatment of PSD and to 
determine treatment based on reliable evidence.

Condition being studied: Post-stroke depression (PSD) is a common complication of stroke, occurs in nearly a third of stroke patients by a study with a five-year follow-up study. In addition, depression often occurs in the first year after stroke, and patients with prior depression or severe stroke are at higher risk for depression. PSD not only increases the suffering of patients, but also imposes a heavy financial burden on families and society.

\section{METHODS}

Participant or population: We will include a systematic review or meta-analysis of patients definitively diagnosed with PSD (including hemorrhagic and ischemic stroke).

Intervention: We will include any systematic review or meta-analysis of nonpharmacological therapy as a trial intervention, including non pharmacological therapy alone or as a complementary alternative to drug therapy.

Comparator: The control group received conventional treatment or placebo. Pharmacological treatment will include selective serotonin reuptake inhibitors (SSRI), serotonin-norepinephrine reuptake inhibitors (SNRI), tricyclic antidepressants and so on.

Study designs to be included: Metaanalyses/systematic reviews based on randomized controlled trials (RCTS) and exclude systematic reviews that did not perform quantitative analysis of the included original studies.

Eligibility criteria: We will include metaanalyses/systematic reviews based on randomized controlled trials (RCTS) and exclude systematic reviews that did not perform quantitative analysis of the included original studies.We will include systematic reviews comparing nonpharmacological therapies with placebo or conventional treatments, and exclude traditional reviews, SR/ meta-analyses in the plan and title stages, conference abstractions, reviews, letters, nonquantitative synthetic meta-analyses, and non-RCT meta-analyses to ensure the accuracy of the final results.

Information sources: We will search for systematic reviews and meta-analyses of the effectiveness of non-pharmacological therapies in treating PSD, We will search the electronic databases MEDLINE, EMBASE, Cochrane Central,Register of Controlled Trials, Web of Science and CINAHL Plus from inception until Octobor 2021.

Main outcome(s): We will include the following outcome measures in systematic review or meta-analysis: Hamilton Depression Rating Scale (HAMD); Selfrating Depression Scale (SDS); National Institute of Health Stroke Scale (NIHSS); Barthel Index (BI); Activities of Daily Living Scale (ADL); Minimum Mental State Examination (MMSE) or any other validated rating scales, which could be linearly transformed to a standardized 100-point scale.

Quality assessment / Risk of bias analysis: Quality of included meta-analyses and systematic reviews will be assessed by AMSTAR. Two researchers will evaluate the quality of studies, and any discrepancies will be resolved by a third author.

Strategy of data synthesis: We will perform various statistical analysis on each eligible meta-analysis with individual study estimates extracted from each metaanalysis. We will calculate the summary effect size, $95 \% \mathrm{Cl}$, and $\mathrm{p}$ value of eligible meta-analysis. Heterogeneity was assessed with the Cochrane's $Q$ test and I 2 statistics.

Subgroup analysis: If the evidence is sufficient, we will conduct a subgroup analysis to determine the difference between different gender and so on. 
Sensitivity analysis: Sensitivity analysis will be carried out to assess the impact of a single study on a comprehensive estimate of each risk factor.

Country(ies) involved: China.

Keywords: non-pharmacological interventions, post-stroke depression, protocol, study, systematic reviews.

Contributions of each author:

Author 1 - Chengcheng Zhang.

Email: zcc13665612780@163.com

Author 2 - Lianyi He.

Author 3 - Zhendong Li.

Author 4 - Hangjian Qiu.

Author 5 - Xiaoqian Wang.

Author 6 - Yuejuan Zhang.

Email: 1171825297@qq.com 